\title{
$10 \mathrm{Gbit} / \mathrm{s}$ Monolithic Integrated MSM-Photodiode AlGaAs/GaAs-HEMT
}

\section{Optoelectronic Receiver}

V. Hurm, J. Rosenzweig, M. Ludwig, W. Benz, R. Osorio, M. Berroth,

A. Hülsmann, G. Kaufel, K. Köhler, B. Raynor, and Jo. Schneider

\author{
Fraunhofer-Institut für Angewandte Festkörperphysik, \\ D-7800 Freiburg, Germany, (Phone: 0761-5159-555)
}

Several research groups have reported on the technology of monolithic integration of MESFETs on GaAs with photodetectors for light of $0.85 \mu \mathrm{m}$ wavelength. In this papex we present the first photoreceiver which is based on a metal-semiconductor-metal (MSM) photodiode and AlGaAs/GaAs HEMTs.

The photoreceiver was fabricated using our established $0.5 \mu \mathrm{m}$ recessed-gate process for double delta-doped quantum well HEMTs /1,2/. The following mean values for the enhancement and depletion HEMT parameters respectively have been obtained: threshold voltage: 0.1 and $-0.5 \mathrm{~V}$, transconductance: 500 and $390 \mathrm{~ms} / \mathrm{mm}$, source resistance: 0.7 and $0.6 \Omega \mathrm{mm}$, transit frequency: 35 and 30 $\mathrm{GHz}$. This process now includes photodiodes. A deep wet etch was used to deposit the photodiodes on an undoped GaAs buffer layer. The $1 \mu \mathrm{m}$ wide photodiode fingers with $1.5 \mu \mathrm{m}$ spacing were defined by electron-beam lithography and subsequent lift-off of Ti/Pt/Au Schottky metal: The DC responsivities of the photodiodes to light of $0.84 \mu \mathrm{m}$ wavelength were $0.25 \mathrm{~A} / \mathrm{W}$ for $4 \mathrm{~V}$ and $0.35 \mathrm{~A} / \mathrm{W}$ for $10 \mathrm{~V}$ bias voltage, respectively. The dark current at $4 \mathrm{~V}$ was less than $2 \mathrm{nA}$ for a photodiode with an active area of $25 \times 25 \mu^{2}$. The monolithic integrated optoelectronic receiver consists of a MSM-photodiode, a transimpedance amplifier, and a $50 \Omega$ output buffer. The transimpedance stage is composed of two enhancement transistors (gate widths $40 \mu \mathrm{m}$ ), two $1 \mathrm{k} \Omega \mathrm{NiCr}$ thin film load resistors, and a $500 \Omega \mathrm{NiCr}$ feedback resistor. The output stage is a source follower with a constant current load (gate widths $80 \mathrm{\mu m}$ ). All high frequency measurements on the receiver were performed on-wafer using CASCADE probes. The photodiode was irradiated by 0.84 $\mu \mathrm{m}$ light from a high speed ORTEL laser diode via a single mode fiber. The current driving the laser diode was modulated to obtain up to $0.8 \mathrm{~mW}$ peakto-peak modulated optical signals. The $-3 \mathrm{~dB}$ bandwidth for sinusoidal modulated incident light lies at $8.2 \mathrm{GHz}$. The circuit response to pulse-modulated non-return-to-zero (NRZ) optical signals was tested at data rates up to $10 \mathrm{GBit} / \mathrm{s}$ using an ANRITSU pulse pattern generator. The eye diagram of the output voltage demonstrates that the optoelectronic receiver operates successfully for a $10 \mathrm{Gbit} / \mathrm{s}$ NRZ pseudorandom data stream of length $2^{7}-1$ bits.

/1/ K. Köhler, P. Ganser, K.H. Bachem, M. Maier, J. Hornung, and A. Hülsmann, Proc. Int. Symp. GaAs and Related Compounds, Jersey, 1990, Inst. Phys. Conf. Ser., 112, p. 521, 1990.

/2/ A. Hülsmann, G. Kaufel, K. Köhler, B. Raynor, K.H. Glorer, E. Olander, B. Weismann, J. Schneider, T. Jakobus, Proc. Int. Symp. GaAs and Related Compounds, Jersey, 1990, Inst. Phys. Conf. Ser., 112, p. 429, 1990. 

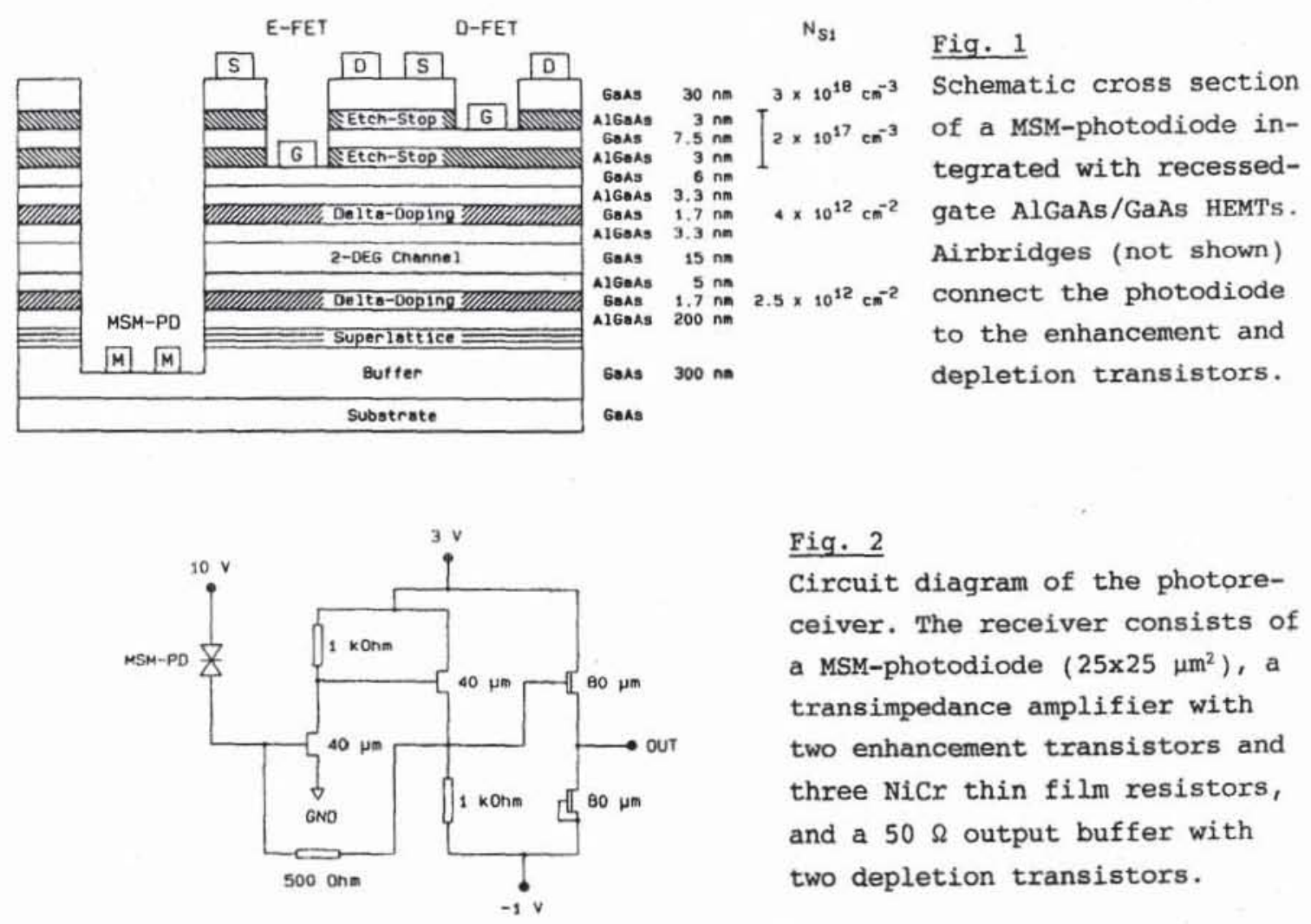

Fig. 2

Circuit diagram of the photoreceiver. The receiver consists of a MSM-photodiode $\left(25 \times 25 \mu \mathrm{m}^{2}\right)$, a transimpedance amplifier with two enhancement transistors and three $\mathrm{NiCr}$ thin film resistors, and a $50 \Omega$ output buffer with two depletion transistors.

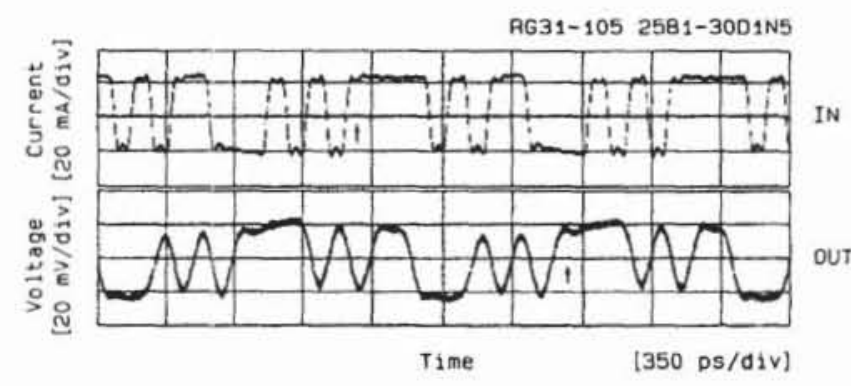

Fig. 3

$10 \mathrm{GBit} / \mathrm{s}$ non-return-to-zero (NRZ) waveforms of the pulse-modulated laser diode current and the photoreceiver output voltage. The time-delay, indicated by the two arrows, is due to the measurement equipment.

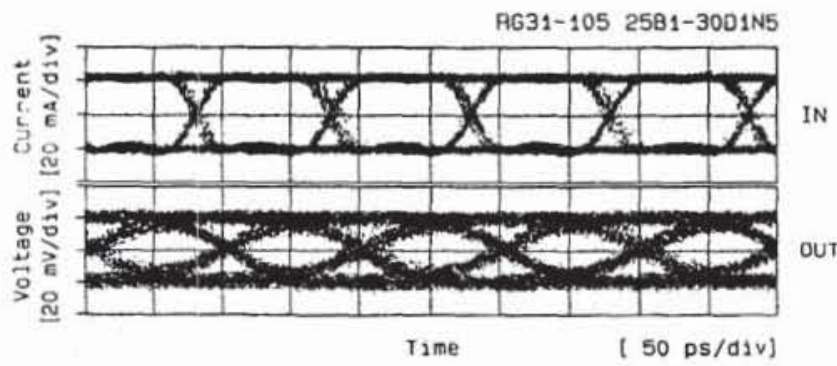

Fig. 4 Eye diagrams of the laser diode current and the photoreceiver output voltage for a $10 \mathrm{GBit} / \mathrm{s}$ NRZ pseudorandom data stream of length $2^{7}-1$ bits. 